Published in : Les Enjeux de l'Information et de la Communication, n¹5/1, 2014, p. 67 à 81

which should be cite to refer to this work.

\title{
Offre numérique en bibliothèque spécialisée : appréhender la perception des usagers via une expérimentation de prêt de dispositifs de lecture nomades, le projet Calliopê
}

\begin{abstract}
Digital resources in a special library: understanding users' perception through a mobile reading devices lending experimentation, the Calliope project
\end{abstract}

Recursos digitales en bibliotecas especializadas: Comprender la percepción de los usuarios a través del experimento de préstamo de dispositivos móviles de lectura. Proyecto Calliope

Article inédit, mis en ligne le 23 novembre 2014

\section{Benoît Epron}

Benoît Epron est docteur en sciences de l'information et de la communication. Il est Maître de conférences et directeur de recherche de l'École nationale supérieure des sciences de l'information et des bibliothèques (ENSSIB) et responsable du Master Publication numérique. benoit.epron@enssib.fr

\section{Stéphanie Pouchot}

Stéphanie Pouchot est docteure en sciences de l'information et de la communication. Elle est professeure en information documentaire à la Haute école de gestion de Genève où elle coordonne le master en information documentaire. Ses recherches portent depuis une quinzaine d'années sur l'organisation, la diffusion et les usages de contenus numériques. stephanie.pouchot@hesge.ch

\section{Hans Dillaerts}

Hans Dillaerts est docteur en sciences de l'information et de la communication. Il est Maître de conférences à l'université Paul-Valéry. Il est associé au laboratoire de recherche LERASS-CERIC.

dillaerts.hans@gmail.com

\section{Philippe Printz}

Philippe Printz est ingénieur d'études en sociologie.philippe.printz@gmail.com

Plan de l'article

Introduction

Lecture sur écran : approches et définition

Contexte de recherche et questionnement

Démarche méthodologique et expérimentation

Analyse thématique des entretiens

Dispositifs et conditions de lecture

Travail ou loisir : des objectifs qui influencent l'activité de lecture

Accès aux offres

Résultats et perspectives

Facilités d'accès, services et repères habituels : des attentes bien définies

Durée et légalité du prêt : des limites importantes

Les expérimentations et analyses en cours

Références bibliographiques 


\section{RESUME}

Au-delà d'un effet de mode lié aux nouveaux dispositifs de lecture, prêter des contenus et des dispositifs numériques répond pour les bibliothèques à un besoin en termes d'accès à l'information. Dans ce contexte, le projet Calliopê vise à appréhender la réception d'une offre de ce type par les usagers, tant au niveau de l'organisation des contenus que des dispositifs prêtés et de leur adéquation aux contenus. Notre questionnement de départ était le suivant: quelle perception globale les usagers ont-ils de ce type d'offre ? Comment s'approprient-ils les contenus ? Existe-t-il des dispositifs privilégiés par type de contenu ? Via une expérimentation de prêts de liseuses et tablettes menée dans une bibliothèque spécialisée en sciences de l'information, nous apportons des éléments de réponse à ces interrogations.

\section{Mots-clés}

Livre numérique, dispositif de lecture, liseuse, tablette, bibliothèque, lecture numérique, offre numérique, prêt

\section{ABSTRACT}

Regardless of passing trends related to new reading devices, lending digital content and devices constitutes for the libraries a response to a need in terms of access to information. In this context, the Calliopê project aims to understand the way patrons receive such an offer, in terms of content organization and the accordance of the borrowed reading devices with the digital content. Our questioning from were as follows: What is the overall perception patrons do have of this type of offer? How do they appropriate the content? Are there privileged devices by content type? Through a lending reading devices (e-book reader, tablet computer) experimentation held in an information and library science library, we provide some answers to these questions.

\section{Keywords}

E-book, reading device, e-book reader, tablet computer, library, digital lecture, digital offer, lending

\section{INTRODUCTION}

Dans le contexte mouvant de l'offre documentaire numérique, les missions et les services proposés par les bibliothèques sont amenés à évoluer. Depuis plus de dix ans, il est ainsi devenu incontournable pour ces institutions d'intégrer la dimension électronique à leur offre documentaire, offre fortement contrainte par les conditions définies par les éditeurs. À la différence des livres imprimés, les conditions de mise à disposition en bibliothèque des livres numériques sont régies en France par des contrats spécifiques à chaque éditeur comme le stipule la loi n²011-590 du 26 mai 2011 relative au prix du livre numérique. La loi autorise en effet les éditeurs à construire des offres sous la forme de licences 
d'utilisation dès lors qu'elles sont « destinées à un usage collectif et proposées dans un but professionnel, de recherche ou d'enseignement supérieur dans le strict cadre des institutions publiques ou privées qui en font l'acquisition pour leurs besoins propres, excluant la revente ». Cela entraîne, de fait, une forte hétérogénéité des modalités d'accès pour les lecteurs.

Au-delà des aspects législatifs et des enjeux économiques que nous n'aborderons pas dans cet article, cet état de fait soulève plusieurs questionnements organisationnels, techniques et d'usages. L'objet du projet Calliopê est de cerner la façon dont les usagers perçoivent et s'approprient une offre numérique globale en bibliothèque spécialisée (ressources éditoriales, interface d'accès et dispositifs de lecture mobile). Ce projet est une réponse scientifique apportée à une problématique professionnelle rencontrée au sein de la bibliothèque de l'enssib dans le cadre de la réflexion sur la mise en place et l'exploitation de son offre et de ses services numériques.

Plus que de la simple mise à disposition de dispositifs, c'est bien du déploiement d'un service dont il est question, appréhendé à la lumière de l'expérience utilisateur dans son ensemble, de l'identification des ressources à leur lecture. L'originalité de notre démarche réside donc dans l'analyse distincte des différentes étapes du processus de lecture numérique.

Dans une première partie de cet article, nous revenons sur les définitions de la lecture numérique en proposant un état de l'art des réflexions et expériences menées à ce jour.

Nous expliquons dans un deuxième temps quelles sont notre problématique et nos hypothèses, puis nous détaillons le déroulement de notre expérimentation. Nous présentons enfin les résultats obtenus et leurs prolongements possibles.

\section{LECTURE SUR ECRAN : APPROCHES ET DEFINITION}

« Nous vivons une longue période de transition culturelle, caractérisée par le reflux de la lecture classique, de la lecture du livre imprimé, non seulement comme savoir et pratique, mais aussi comme référence, comme référence principale, unificatrice, et seule dans cette fonction unificatrice, pour l'accès à la culture et à la citoyenneté »(Giffard, 2010).

La pratique de la lecture est intimement liée au texte qui constitue un objet matériel et singulier engagé dans un processus pratique de communication (Jeanneret, 2001; Davallon et al., 2003). Comme le souligne Yves Jeanneret, "l'objet-texte est toujours un objet complexe sur le plan sémiotique, relevant de plusieurs systèmes de signes reliés les uns aux autres en une configuration. En particulier, le texte écrit combine un ensemble composite de codes, associés dans un espace «scripto-visuel», ce qui signifie que l'ensemble des signes qu'il donne à déchiffrer sont régis comme en surplomb par un système signifiant plus général qui définit l'«image du texte », l'organisation des espaces de lecture. » (Jeanneret, 2001)

Lire, c'est mettre en œuvre des compétences diverses. Claire Bélisle (Bélisle, 2002) en distingue quatre : la compétence linguistique (mobilisation d'un ensemble de 
connaissances linguistiques), la compétence encyclopédique (mobilisation d'un ensemble de savoirs), la compétence logique (capacité de raisonner) et enfin la compétence rhétorique-pragmatique ou discursive (application de savoirs sur les principes discursifs). La pratique de la lecture s'inscrit toujours dans un contexte socio-culturel donné qui fait figure de contrat de lecture (Bélisle, 2002). Ce contrat «permet de définir un cadre de référence commun entre les auteurs et les lecteurs. Il est acquis par l'intériorisation des régularités textuelles auxquelles sont confrontées les diverses expériences de lecture: genre, disposition, schéma de récit. » (Bélisle, 2002)

Selon Terje Hillesund, la pratique de la lecture est marquée par des degrés divers de continuité, temporalité, spatialité et de "connectivité thématique » (Hillesund, 2002) selon les contextes et objectifs du lecteur. L'auteur distingue également la lecture continue spatiale, soit une lecture linéaire, et la lecture discontinue, c'est-à-dire une lecture non linéaire qui peut également être qualifiée de «lecture fragmentée». Ces deux modalités de lecture ne sont pas exclusives, au contraire, il est fréquent qu'elles soient combinées, notamment dans des pratiques de lecture d'articles en ligne (Hillesund, 2002).

Les questions liées à la lecture numérique intéressent depuis plusieurs années à la fois la communauté professionnelle des bibliothèques (bibliothèques universitaires et bibliothèques de lecture publique) et les chercheurs travaillant sur les dispositifs de lecture numérique (Bélisle, 2002 ; Hillesund, 2002 ; Limam et al., 2008 ; Giffard, 2009 ; Zamuner, 2012 ; Cabot, 2013; Hulin, 2013). Dans le cadre de cette réflexion, de nombreux établissements français mettent actuellement à disposition de leurs lecteurs des terminaux adéquats $^{1}$. Ce sont surtout «les bibliothèques publiques [qui] ont toutefois commencé à acquérir et prêter des liseuses et des tablettes électroniques. La bibliothèque publique devient ainsi progressivement un lieu de découverte de ces nouveaux outils de lecture. » (Dillaerts, Epron, 2014. Ces dispositifs de lecture sont la plupart du temps préchargés avec des livres tombés dans le domaine public et il s'agit souvent de prêts à court terme. Ces initiatives donnent lieu le plus souvent à des comptes rendus d'expériences dans la littérature professionnelle. Par exemple, David Liziard (Liziard, 2011) recense chronologiquement les étapes du projet ayant abouti à la mise en place du prêt de liseuses aux médiathèques d'Issy-les-Moulineaux. Ce retour est intéressant, car il pointe pour chaque phase (initiation du projet, choix des modèles, aspects techniques, appropriation par l'équipe, tests, sélection et préchargement des textes, lancement) les aspects sur lesquels être vigilant. La nécessité de prendre en compte le contexte, notamment financier et stratégique, est également soulignée, de même que l'importance des aspects humains, tant du côté des professionnels en poste que des lecteurs. En outre, évidence et simplicité sont également pointées comme étant les facteurs clefs du succès de tels services.

De fait, ces écrits ont pour principal objectif un partage des connaissances dans une communauté de pratiques : ils constituent à ce titre de précieux témoignages et mobilisent in fine assez peu d'approches théoriques. Par ailleurs, les cadres méthodologiques déployés

${ }^{1}$ Une carte recensant les établissements prêtant des dispositifs mobiles de lecture en France a été initiée en 2012. Elle comporte aujourd'hui plus de 400 établissements [page consultée le 4 juillet 2014] https://maps.google.fr/maps/ms?msid=217057881769664868980.0004c29912fbdd0cb822e\&msa=0 
restent souvent relativement flous. En outre, c'est généralement un seul aspect qui est traité : le prêt de dispositifs, l'offre de livres numériques ou la mise en place d'un outil de découverte intégré au système informatique de gestion de la bibliothèque.

Nous proposons ici d'aller plus loin en envisageant l'ensemble du processus : la mise à disposition de dispositifs de prêt numérique (contenus et outils de lecture numérique) tout d'abord, et son usage par les lecteurs ensuite. Cela nous permet également d'analyser dans quelle mesure les dispositifs de lecture sont en adéquation avec l'offre documentaire et la façon dont l'organisation de celle-ci est perçue.

\section{CONTEXTE DE RECHERCHE ET QUESTIONNEMENT}

Notre questionnement initial concernait la mise en place par la bibliothèque de l'enssib d'un nouveau service accompagnant le développement de son offre de livres numériques. Plus précisément, la question posée par la bibliothèque de l'enssib au département de la recherche, portait sur un service de prêt de dispositifs mobiles de lecture numérique qui soit en adéquation avec son offre documentaire.

Une hypothèse largement répandue dans le domaine des bibliothèques est celle d'un usage limité des ressources numériques en raison d'un équipement des usagers peu adapté à la lecture numérique de textes longs. Cette hypothèse s'accompagne d'une attention particulière accordée par les professionnels des bibliothèques à l'accessibilité non discriminée à leurs ressources. Préoccupation fréquente lorsqu'elle concerne les services documentaires analogiques, elle prend une dimension plus complexe lorsqu'il s'agit de lisser les évidentes disparités en termes d'équipement des publics.

Dans ce contexte, la première solution évidente semble être de prêter des dispositifs permettant la lecture des livres numériques. Il s'agit ici d'une réponse cohérente, le prêt étant une activité traditionnelle des bibliothèques. La matérialité des dispositifs permet de plus de calquer ce système de prêt sur les autres objets (livres, revues...) proposés en bibliothèque ; d'où un questionnement très pragmatique sur le type de dispositif à proposer.

Afin d'y répondre, nous avons déployé une expérimentation basée sur le prêt de dispositifs de lecture numérique variés, articulée à une offre de ressources éditoriales numériques, l'objectif étant d'identifier et mesurer une adéquation éventuelle entre un type de ressources et un type de dispositif. Notre objectif était de définir des typologies regroupant des types de ressources éditoriales et des dispositifs de lecture.

Plus généralement, le projet visait à répondre au questionnement suivant : quelle perception les usagers ont-ils de ce type d'offre ? Comment s'approprient-ils les contenus ? Existe-t-il des dispositifs privilégiés par type de contenu ? Le prêt de tels dispositifs est-il pertinent?

Cette problématique a évolué au cours de notre étude et s'est ainsi enrichie d'aspects relatifs à l'expérience utilisateur et aux attentes, besoins et perceptions des usagers. Il s'est 
notamment agi de comprendre la manière dont ils s'approprient les dispositifs de lecture mobile prêtés par une bibliothèque. Un des objectifs de ce travail est donc également d'évaluer dans quelle mesure les équipements constituent des freins dans l'utilisation ou non des ressources électroniques proposées par la bibliothèque de l'enssib.

\section{DEMARCHE METHODOLOGIQUE ET EXPERIMENTATION}

L'enssib est l'établissement supérieur chargé, entre autres, de la formation des cadres des bibliothèques françaises. Sa bibliothèque constitue de fait un terrain d'expérimentation particulier puisqu'elle accueille un public spécialisé en sciences de l'information et des bibliothèques. Afin de mieux connaître l'environnement numérique et documentaire des 524 inscrits à l'époque (en 2012) ainsi que leurs pratiques numériques, nous avons mené une enquête préalable de type quantitatif via un questionnaire en ligne et sa version papier dans les locaux de la bibliothèque. Cent cinquante et une personnes ont répondu à l'enquête sur la base du volontariat. Il s'agit d'individus relativement connectés à Internet et attentifs aux outils choisis pour satisfaire leurs besoins d'information. Les deux tiers des répondants ont entre 20 et 39 ans. Ce faisant, ils ne s'entourent pas d'une multitude de dispositifs ou d'applications pour y parvenir : ils choisissent contextuellement l'outil qui leur paraît le mieux à même de satisfaire leurs besoins. Aussi, la tablette, la liseuse et le smartphone ne sont pas des équipements très utilisés à la bibliothèque.

En revanche, les ordinateurs fixes et portables sont incontournables, aussi bien dans le monde du travail que de la sphère privée. La population étudiée dans le cadre de cette expérimentation présente un niveau d'accès potentiel aux ressources numériques relativement élevé puisqu'ils disposent à 93\% d'une connexion internet à leur domicile, que deux tiers d'entre eux passent plus de $10 \mathrm{~h}$ par semaine devant leur ordinateur et que plus de $80 \%$ ont un ordinateur portable. Le développement de services documentaires dématérialisés semble donc pertinent, car la tendance forte des usages des répondants de cette enquête apparaît compatible avec une offre de ressources documentaires numériques spécialisées.

Bénéficiant d'une connaissance plus précise de l'équipement des publics de la bibliothèque, nous avons dans un deuxième temps adopté une démarche qualitative de manière à cerner plus finement les besoins, attentes et ressentis des usagers. La complémentarité des deux approches est aujourd'hui avérée et permet d'avoir une vision, sinon exhaustive, tout du moins, globale de la situation.

Pour cette seconde phase de l'étude, nous souhaitions confronter des lecteurs à une offre numérique globale, c'est-à-dire proposant des terminaux nomades de lecture d'une part (différents modèles de tablettes et liseuses à encre électronique en complément de leur propre matériel) et un ensemble organisé de ressources documentaires numériques d'autre part. Parmi l'éventail méthodologique en recherche qualitative, nous avons opté pour le focus group. Du fait du dialogue engagé entre les participants, le recueil de données effectué dans ce contexte permet d'obtenir des éléments riches (retours individuels) et confrontant différents points de vue. 


\section{Constitution du panel}

Concomitamment à l'enquête quantitative mentionnée plus haut, un appel à volontaires a été lancé via le site web de l'enssib pour participer à une expérimentation sur l'offre numérique à la bibliothèque.

Les critères de participation étaient les suivants :

- être inscrit à la bibliothèque de l'enssib et avoir un numéro d'abonné en cours de validité ;

- disposer d'un dispositif mobile personnel de type ordinateur portable ou smartphone;

- être disponible aux dates de réunion prévues et s'engager à se déplacer à l'enssib pour y participer.

\section{Déroulement}

Il a été demandé aux participants d'être présents lors de quatre rendez-vous à l'enssib : une réunion de lancement, deux réunions intermédiaires et une réunion de clôture.

Suite aux candidatures, nous avons sélectionné quinze volontaires répondant aux critères énoncés ci-dessus, âgés de 22 à 46 ans et offrant un panel de profils variés (étudiants, bibliothécaires, personnel administratif et académique). Chacun d'entre eux a testé successivement trois dispositifs de natures et marques différentes (tablette, liseuse et dispositif personnel) durant trois sessions de 3 semaines (durée du prêt à la bibliothèque de l'enssib). L'ordre d'utilisation des dispositifs variait selon les participants de manière à limiter leur influence éventuelle sur la façon d'appréhender l'offre et les supports euxmêmes.

Une réunion de lancement a permis de présenter les objectifs de l'étude aux participants et son déroulement (utilisation des dispositifs, réunions intermédiaires, changements de dispositifs) ainsi que l'étagère numérique. À cette occasion, l'équipe de recherche a également sensibilisé les participants à la question de l'environnement de lecture numérique (formats, transferts, dispositifs) en leur communiquant le guide édité sur le sujet par la bibliothèque de l'enssib?2.

Trois autres rencontres ont eu lieu dont le déroulement était le suivant :

- en parallèle, trois focus group de cinq participants animés par l'équipe de recherche et enregistrés ;

- un repas pris en commun et discussion informelle sur la phase précédente ;

- retour des dispositifs testés et, le cas échéant, emprunt du nouveau matériel à tester.

Pour des raisons organisationnelles, les répondants ont été répartis en deux focus groups lors de la dernière rencontre. Nous avons ainsi colligé les enregistrements de huit focus groups.

\section{Traitement des données}

Un peu moins de sept heures de discussion ont été enregistrées puis retranscrites. Nous avons ainsi travaillé pour ce volet qualitatif sur un corpus de 88684 mots. Afin d'analyser ces données, nous avons opté d'une part pour une analyse assistée par ordinateur et

\footnotetext{
${ }^{2}$ Version en ligne disponible sur http://www.enssib.fr/sites/www/files/Bibliotheque/guide_dispositifs_de_lecture-v2.pdf [page consultée le 04 juillet 2014]
} 
d'autre part pour une analyse de ce corpus de manière plus classique, suivant un passage en revue manuel et une lecture approfondie des textes de façon à en extraire les thématiques principales et les éléments saillants.

La partie suivante présente nos principaux résultats selon les étapes de l'expérience des utilisateurs, de la prise en main de l'appareil à l'exploitation des contenus en passant par la découverte de l'organisation de ces contenus et les aléas techniques de leur téléchargement.

\section{ANALYSE THEMATIQUE DES ENTRETIENS}

\section{Dispositifs et conditions de lecture}

Les répondants emploient un vocabulaire plus pratique qu'émotionnel à l'égard du matériel testé : «la taille de l'écran est trop grande »... De l'ordre du ressenti, ils utilisent surtout les termes « agréable » et «confortable», aussi bien positivement que négativement.

Cependant, l'appréciation du matériel est globalement négative. Même si les répondants reconnaissent parfois qu'un appareil peut être agréable et plaisant au premier abord, dès lors que l'utilisation est plus poussée le discours devient de plus en plus critique. Nous pouvons distinguer dans un premier temps un effet de nouveauté enthousiasmant et, dans un second temps, une comparaison avec le papier, entraînant des jugements négatifs. Les interviewés insistent sur des aspects techniques : taille de l'écran, luminosité, connectivité et interface.

L'élément saillant de ces discussions est la perception d'une certaine adéquation entre les différents types de lecture et les dispositifs. Pour des textes courts (articles de journaux, blogs, etc.), la plupart des participants accepte l'utilisation d'une tablette ou une liseuse. Pour la majorité des interrogés, la liseuse est perçue comme un terminal plus propice à une lecture longue que la tablette : plus légère, de l'encre électronique, pas de rétro-éclairage, une taille d'écran au format d'un roman de poche, plus confortable... Tous ces éléments font de la liseuse un dispositif déclaré comme plus adapté que la tablette à une lecture en profondeur. En pratique, les candidats restent toutefois attachés au papier pour des lectures plus longues.

«(...) en fait, j’ai du mal quand même à lire des formes longues sur le numérique, ça c'est clair. »

Le confort de lecture sur une tablette, liseuse ou ordinateur portable dépend donc du temps passé sur l'appareil et de la longueur des textes. La tablette, du fait de son caractère connecté et multimédia, est pour certains un dispositif inadapté à une lecture longue, car sa polyvalence (navigation web, jeux, etc.) détourne vite l'attention. D'autres, au contraire, valorisent cette connexion qui permet la recherche rapide d'informations complémentaires lors d'une lecture. Le manque de connectivité de certaines liseuses et, le cas échéant, la difficulté à accéder aux ressources en ligne sont par ailleurs déplorés. Une fois la liseuse prête à l'emploi, ces lacunes constituent toutefois un atout lors de la lecture pour la majorité des participants :

"C'est l'avantage de la liseuse d'être déconnectée "

"Mais le problème, c'est que cette connexion, du coup, elle entraîne très vite une pollution dans la lecture...» 
Par ailleurs, le rétro-éclairage des tablettes est critiqué par la quasi-totalité des interviewés (trop lumineux, brillant, problème de reflets...). De plus, leur poids rend inconfortable son utilisation en situation de lecture longue. Ces résultats coïncident avec les résultats publiés par Terje Hillesund concernant la lecture sur écran d'ordinateur (Hillesund, 2010).

En revanche, la tablette semble particulièrement adaptée pour des lectures courtes comme pour celle de livres illustrés et de bandes dessinées. Le rendu des couleurs des tablettes (surtout celui de l'iPad) est très apprécié par les interrogés.

L'esthétique de l'appareil revêt en outre une certaine importance pour les répondants : plus le dispositif est considéré comme "beau », plus la lecture est envisagée. Ainsi, l'appréhension de l'ouvrage numérique dépend en partie de l'image perçue de l'objet qui permet d'y accéder. L'expérience de lecture numérique est, comme pour le papier, influencée par l'objet et le contenu : il est donc préférable de parler ici d'incitation à la lecture plutôt que de plaisir de lecture. En outre, les liseuses sont moins bien perçues que les tablettes, comme le montrent les qualificatifs employés à leur sujet ("sombre», " cheap ", " y'a un côté moins glossy, objet cher, objet de luxe»). Selon Margit Molnar (Molnar, 2012) qui définit la pratique de la lecture comme une articulation simultanée du geste corporel (le ressenti par nos organes sensoriels) et du geste mental (apport intellectuel du texte), l'objet «esthético - technologique » joue en effet un rôle central dans la lecture numérique. Elle souligne ainsi : «Dans le cas du livre imprimé, le texte fait corps avec son support sur lequel il est imprimé, il s'identifie à celui-ci. Le texte numérique change de « corps » chaque fois où il apparaît sur l'écran d'un autre objet. [...] Ainsi la variété des informations sensorielles directes se réfère à l'objet qui est, pour ainsi, détaché du contenu textuel. A nos yeux, ce détachement de l'objet - support et de l'écrit joue un rôle primordial dans la réception du texte et donc dans la pratique de lecture et dans le rapport à cette pratique en général car les qualités sensorielles propres à chaque livre imprimé, dans le cas de la lecture numérique sont éliminées, sinon uniformisées au goût de l'objet technologique utilisé. C'est ainsi que l'objet "esthético - technologique » se trouve au cœur de nos préoccupations. ».

À ce sujet, précisons que les entretiens nous révèlent également que la fragilité dégagée par les dispositifs ainsi que leur valeur marchande peuvent constituer des freins à la fois pour l'usage de l'outil et, conséquemment, la lecture numérique.

«(...) puisque je l'ai pas pris dans les transports de ville mais euh...j'étais dans le TER, j’allais dans une région reculée y'avait personne, on sort l'iPad y'a aucun problème. "

"Une liseuse, c'est moins ostentatoire », "À la plage, le sable peut s’infiltrer dedans alors qu'un livre on ne se pose pas la question».

Globalement, les participants n'utilisent pas de dispositifs numériques dans les transports en commun. Il s'agissait en effet d'un matériel qui leur était prêté et ils ont évoqué leur crainte de se le faire voler ou de l'endommager. Nous pensons toutefois que ces craintes existeraient également s'il s'agissait de dispositifs personnels. Les dispositifs de lecture numériques représentent un investissement financier certain qui incite naturellement à la prudence. 


\section{Travail ou loisir : des objectifs qui influencent l'activité de lecture}

Les participants exercent en grande partie leur activité professionnelle devant un ordinateur et ne manifestent pas l'envie de retrouver un dispositif numérique en dehors du cadre professionnel, surtout les plus âgés d'entre eux.

"...et en plus comme moi je travaille sur un écran huit heures par jour, j'ai pas forcément envie de lire sur un écran une fois chez moi donc je lis volontiers les articles courts, de la presse, des PDF, etc. »

Notons qu'ils alternent deux types de lecture : professionnelle et "plaisir ». L'ordinateur est considéré avant tout comme un outil de travail alors que la liseuse et la tablette ont une dimension loisir, même si cette dernière peut occasionnellement servir à la lecture de fichiers PDF liés au travail.

«Pour moi lire sur un écran, c'est lié, soit au travail, soit à la recherche d’information»

La problématique de l'annotation comme celle du surlignage ont été évoquées à plusieurs reprises par les participants. Lorsque les lectures nécessitent d'être annotées, le papier est sans conteste le support privilégié. Certains participants mettent en avant la difficulté de prendre des notes sur une tablette ou même un ordinateur. D'autres, en revanche, se sont approprié cette forme d'écriture qui devient opératoire sur des ouvrages empruntés, car elle en respecte l'intégrité :

«Je me suis découvert une forme d'appétence au surlignage numérique, parce que je peux l'enlever quand je veux aussi. »

Finalement, un tiers des participants trouvent ces fonctionnalités intéressantes, sans les utiliser pour autant, ce qui s'explique probablement par le fait que les conditions d'accès proposées à l'époque par la bibliothèque de l'enssib, notamment le streaming, ne permettent pas de conserver les annotations de manière pérenne.

Par ailleurs, si leur environnement professionnel leur fournit du matériel informatique prêt à l'emploi, le fait de devoir s'approprier de façon autonome une tablette ou une liseuse et l'environnement logiciel afférent peut s'avérer décourageant. En effet, pour la moitié des participants, la manipulation de ces dispositifs reste compliquée. Toutefois, du fait de leur appartenance au monde de la bibliothéconomie et des sciences de l'information, ils ont su développer des stratégies leur permettant finalement de compenser leurs lacunes, en consultant par exemple des forums et des aides en ligne.

\section{Accès aux offres}

Les participants éprouvent des difficultés à appréhender les infrastructures d'accès aux ressources numériques (connectivité, interfaces, compatibilité logicielle et matérielle). Ainsi, l'étagère numérique proposée par la bibliothèque de l'enssib est perçue dans un continuum rassemblant d'autres plateformes de mise à disposition de ressources numériques textuelles (gratuites ou payantes). De ce fait, elle est largement comparée à d'autres offres. Les principales critiques portent alors sur deux aspects : d'une part, une organisation inadaptée des contenus, d'autre part, une ergonomie peu satisfaisante.

Par ailleurs, l'impossibilité d'accéder directement aux ressources depuis une liseuse (recours quasi-systématique à un processus fastidieux nécessitant de passer par un ordinateur connecté) accroît les difficultés perçues et l'insatisfaction des répondants. 
«Du coup accéder directement à l'étagère numérique en n'utilisant que la liseuse, ça relève de, enfin ça relève de l'acte de conviction... hein... c'est pas... voilà... donc ça veut dire que la liseuse ne peut pas réellement être utilisée... sans un ordinateur. »

Les mécanismes d'authentification pour rejoindre le réseau de la bibliothèque depuis l'extérieur de l'enssib alourdissent également la démarche: quel que soit le dispositif utilisé, la très grande majorité des participants a éprouvé d'importantes et nombreuses difficultés à se connecter à l'étagère numérique, mais aussi à n'importe quelle autre plateforme éditoriale proposée par l'enssib. L'obligation de passer par un VPN est trop contraignante pour les usagers les moins à l'aise techniquement: ce processus d'authentification est perçu comme "lourd", "pas convivial», "ne fonctionne pas ». Ces participants se découragent facilement et abandonnent donc les démarches : "Moi j'avoue que c'est vrai que du coup j'ai pas fait d'effort non plus », "Moi j'ai pas trop insisté non plus »

A l'inverse, les individus pourvus d'un capital technique plus développé mettent quant à eux en place des stratégies alternatives permettant d'accéder aux ressources souhaitées : " bidouillage », « bricolage ».

Dans les deux cas, la démarche est vécue comme «laborieuse».

Ces conclusions rejoignent l'un des constats du rapport Ayushi, étude française portant sur les usages et les besoins d'étudiants de premier cycle et d'enseignants en matière de ressources numériques. L'étude souligne en effet que : "Compte tenu du niveau d'équipement élevé des étudiants et de leur forte propension à être connectés à Internet, nous pourrions penser qu'ils ont intégré les mécanismes inhérents au développement du numérique. Au contraire, ils sous-utilisent les outils qui sont à leur disposition. Leurs connaissances se limitent donc à la navigation dans un moteur de recherche ainsi qu'à la fréquentation de réseaux sociaux. L'habitude de ces plates-formes user friendly masque un manque de compétences et freine leur pleine appropriation des outils numériques. » (Printz, 2012)

Une fois passée cette barrière d'accès aux plates-formes, d'autres difficultés apparaissent : l'accès aux ressources ainsi que le format des fichiers. Rappelons que les interviewés sont des professionnels des bibliothèques, ou en devenir, ils sont donc familiers avec la recherche d'information et la navigation. Ils déplorent ici :

- l'architecture de l'offre rassemblée sur l'étagère numérique, proposée de manière thématique alors qu'il est attendu une organisation rappelant la bibliothèque physique ;

- le chemin d'accès aux ressources identifiées, considéré comme désordonné ;

- la multiplicité des formats de fichiers et leur incompatibilité avec certains dispositifs de lecture.

\section{RESULTATS ET PERSPECTIVES}

Facilité d'accès, services et repères habituels : des attentes bien définies

Il semble vain de vouloir construire une offre de dispositifs sur la base de stéréotypes de lecteurs qui semblent en réalité délicats à cerner et définir. En effet, même si de grandes 
tendances se dégagent, les pratiques et perceptions sont propres à chaque usager, même pour un public spécialisé. Ainsi, il n’y pas de «bon dispositif » par défaut.

L'originalité de notre étude est de mettre en évidence à la fois la diversité des modalités de lecture numérique et la difficulté de faire émerger des typologies précises de combinaisons pratiques-dispositifs-contenus. Par conséquent, il est primordial que les bibliothèques définissent des services numériques compatibles avec la diversité des pratiques des usagers. Jérôme Dinet (Dinet, 2009) souligne pourtant l'importance d'une conception centrée sur les utilisateurs pour les bibliothèques numériques. Nous suggérons en ce sens l'exploitation des potentialités offertes par le numérique en termes d'individualisation des interfaces et des services. Pour Le Coadic, «Toutes les décisions sont alors prises dans un cadre institutionnel qui place l'usager en position centrale, qu'elles concernent la planification, les opérations ou la gestion» (Le Coadic, 2004).

Bien qu'issues d'une expérimentation en bibliothèque spécialisée, dans un environnement académique, nos conclusions rejoignent les travaux concernant la lecture publique menés par Kani-Zabihi et al. (Kani-Zabihi et al., 2008) auxquels se réfère Dinet lorsqu'il dresse l'inventaire des principales attentes des usagers des bibliothèques. Premièrement, "les contenus doivent être facilement accessibles et ne pas exiger de compétences techniques ou de connaissances en documentation particulières» (Dinet, 2009). Dans notre cas, les problématiques de transfert de fichiers et leur compatibilité avec les dispositifs de lecture autant que l'authentification paraissent rédhibitoires. Les usagers sollicitent des accès facilités aux différentes plates-formes éditoriales notamment via une identification unique.

Deuxièmement, «une bibliothèque numérique doit offrir les mêmes services qu'une bibliothèque traditionnelle» (Dinet, 2009). Nos interviewés soulignent cette perception comparée des offres numériques et traditionnelles en pointant la nécessité d'une organisation intellectuelle des collections pertinente. Plus précisément, l'attente des usagers porte sur une unification des différentes offres au sein d'un plan de classement unique.

Enfin, «une bibliothèque numérique doit être conçue de manière à ce que l'utilisateur retrouve ses «marques» telles que l'obtention de l'aide d'employés» (Dinet, 2009). L'attente de nos testeurs par rapport à l'expertise des personnels des bibliothèques est forte. Elle porte sur une organisation raisonnée des ressources, mais aussi sur la capacité d'informer (voire de former) et de conseiller de façon neutre, tant sur les dispositifs de lecture numériques ou que sur l'offre documentaire. Il peut également s'agir d'aider le lecteur habitué aux rayons de sa bibliothèque physique à se repérer plus facilement et à trouver les ressources auxquelles il souhaite accéder (via par exemple la création de dossiers thématiques, la mise en place d'étagères virtuelles personnalisables, la réutilisation des photos des couvertures sur l'interface ou encore la présentation par type de support).

\section{Durée et légalité du prêt : des limites importantes}

On l'a vu, dans le domaine du numérique en bibliothèque, les services attendus sont similaires à ceux offerts traditionnellement. Pour autant, la transposition telle quelle de tous les services montre rapidement ses limites de faisabilité et de pertinence : le prêt de courte durée, inspiré des durées de prêts des ouvrages papier, apparaît par exemple comme une limite importante à l'appropriation des dispositifs de lecture par les usagers et, de ce fait, à l'usage des ressources numériques. Cela soulève la question de prêts à plus long 
terme, dont la durée serait calée sur le calendrier académique (semestre, année). Cette proposition suppose que les bibliothèques soient en mesure de proposer ce type de prêt c'est-à-dire qu'elles lèvent les obstacles liés au financement de l'investissement initial, à l'évolution rapide des dispositifs et des technologies ainsi qu'à la gestion des dispositifs en eux-mêmes (configuration, suppression des données utilisateurs).

Le probable frein constitué par les aspects financiers d'un prêt de longue durée laisse ouvertes des opportunités pour un renforcement du rôle des professionnels des bibliothèques sur deux plans, via un positionnement :

- en tant qu'expert, prescripteur sur les dispositifs, dans le respect d'une neutralité et d'une objectivité reconnues à des agents du service publique. Cette activité de conseil peut s'incarner dans les établissements par la mise en place d'espaces de démonstrations et de tests, comme à la bibliothèque des Champs Libres à Rennes ou, finalement, celui qui a été mis en place à la bibliothèque de l'enssib.

- en tant que professionnels capables de déployer des architectures centralisées regroupant l'ensemble de leur offre dans une proposition documentaire unique et correspondant au public de la bibliothèque.

Pour assurer au mieux ces missions, il est nécessaire pour les agents d'être dans une dynamique de veille, de formation et/ou d'autoformation.

Notons en outre le flou du cadre juridique sur le prêt de liseuses et de tablettes en bibliothèques. Pour le moment, cette pratique semble être tolérée par les fabricants dans la mesure où peu de dispositifs de lecture numériques sont proposés à l'emprunt. La mise en place de services de prêt à grande échelle et à long terme risque toutefois de changer la donne. Lionel Maurel souligne ainsi que les bibliothèques prêtant des liseuses à leurs lecteurs «n'avaient pas d'autre recours que d'acheter les appareils comme l'aurait fait un particulier. Ce faisant, ont-elles agi de manière légale ? Ce n'est pas certain. La question a été examinée par certains professionnels américains, qui ont relevé que les conditions d'utilisation des appareils contenaient des limitations à l'usage personnel ou privé. Dès lors, on est en droit de se demander si les tablettes sont bien bibliocompatibles...» (Maurel, 2011).

\section{Les expérimentations et analyses en cours}

Rappelons que les conclusions présentées dans cet article relèvent d'un terrain particulier, celui d'une bibliothèque spécialisée en sciences de l'information. Nous avons souhaité élargir notre expérimentation à d'autres environnements. Compte tenu des différences contextuelles relatives à la nature des offres, aux modèles économiques afférents et aux spécificités des publics, nous avons déployé l'expérimentation au sein du SCD de l'Université Lyon 1 et de la Bibliothèque Municipale de Lyon. Enfin, un élargissement de l'étude à la Suisse romande est en cours depuis janvier 2014. Le croisement de l'ensemble de ces résultats nous permettra de dresser un panorama plus complet sur cette question. 


\section{REFERENCES BIBLIOGRAPHIQUES}

Belisle, Claire (2002), "Lire avec un livre électronique : un nouveau contrat de lecture ?", In : Les défis de la publication sur le web: Hyperlectures, cybertextes et méta-éditions. Colloque dans le cadre des "Quinzièmes entretiens" du Centre Jacques Cartier, Lyon, 9-11 décembre 2002, http://archivesic.ccsd.cnrs.fr/sic 00000422/.

Cabot Auriane (2013), Les pratiques de lecture et le numérique : l'intégration de la lecture numérique dans le quotidien. Mémoire de Séminaire : Pratiques communicationnelles et numérique, Université Lyon 2.

Davallon, Jean, Despres-Lonnet, Marie, Jeanneret, Yves, Le Marec, Joëlle, Souchier, Emmanuel (2003), «Introduction », In : Davallon, Jean, Despres-Lonnet, Marie, Jeanneret, Yves, Le Marec, Joëlle, Souchier, Emmanuel. Lire, écrire, récrire : Objets, signes et pratiques des médias informatisé. Éditions de la Bibliothèque publique d'information.

Dillaerts, Hans, Epron, Benoît (2014), «La place du livre numérique dans les bibliothèques publiques françaises: État des lieux et prospective». Revue canadienne des sciences de l'information et de bibliothéconomie, 2014, vol. 38 (2), p. 80-96.

Dinet, Jérôme (2009), "Pour une conception centrée-utilisateurs des bibliothèques numériques ». Communication E langages, 2009, vol. 2009, n¹61, p 59-74.

Giffard, Alain (2009), "Les lectures industrielles». In : Stiegler Bernard, Giffard Alain, Faure Christian. Pour en finir avec la méconnaissance : Quelques réflexions d'Ars Industrialis. Paris : Flammarion

Giffard, Alain (2010), "Lecture numérique et culture écrite». Revue skhole.fr, http://skhole.fr/lecture-num\%C3\%A9rique-et-culture-\%C3\%A9crite-par-alain-giffard

Hillesund, Terje (2010), " Digital reading spaces : How expert readers handle books, the Web and electronic paper ». First Monday, april 2010, vol. 15, n 4

Hulin, Timothée (2013), Terminaux mobiles et bibliothèques : quelles opportunités, quelles perspectives ?. Mémoire DCB, enssib.

Jeanneret, Yves (2001), «Informatic literacy : manifestations, captations et déceptions dans le texte informatisé ». SPIRALE - Revue de Recherches en Éducation, $\mathrm{N}^{\circ} 28$

Kanie-Zabihi E., Ghniea G., Chen S.Y. (2008), « User perceptions of online public library catalogues », International Journal of Information Management, vol.28, ${ }^{\circ} 6$

Le Coadic, Yves F. (2004), Usages et usagers de l'information. Paris : ADBS

Limam Latifa, Hachicha Sami, Bsir Besma, Romdhane Mohamed Ben, Mkadmi Abderrazak (2008), «Les pratiques de la lecture numérique : cas des enseignants chercheurs tunisiens », In : Colloque international franco-tunisien SFSIC/ISD/IPSI. Interagir et transmettre, informer et communiquer : quelles valeurs, quelle valorisation, Tunis, 17-18-19 avril 2008.

Liziard, David (2011), "Prêter des liseuses électroniques : un projet en 7 étapes ». In : Jacquinet Marie-Christine. Créer des services innovants. Stratégies et répertoire d'actions pour les bibliothèques. Villeurbanne : Presses de l'enssib, pp. 81-87.

Maurel, Lionel (2011), «Faut-il respecter le droit en bibliothèque ?». Bulletin des bibliothèques de France, 2011, n 3, http://bbf.enssib.fr/consulter/bbf-2011-03-0006-001.

Molnar, Margit (2012), "Lecture numérique, entre faire et dire, la complexité du geste intuitif », Université d'été Ludovia, Ax-Les Thermes, 27-30 août 2012, Actes publiés en ligne, http://culture.numerique.free.fr/publications/ludo12/molnar ludovia 2012.pdf. 
Printz, Philippe (2012), Étude des usages et besoins concernant l'utilisation d'outils et de ressources numériques.Villeurbanne : enssib,

http://www.enssib.fr/sites/www/files/documents/recherche/rapport-ayushi-nov2012.pdf

Zamuner, Emeline (2012), Réalités et perspectives de la lecture numérique en bibliothèque, un panorama de la lecture publique à la recherche universitaire. Mémoire de Master. Université Stendhal. 